excluded before considering this diagnosis. It is difficult to prove that a malignant melanoma has its primary point of origin in internal organs because the possibility exists that it is a metastasis from a small primary tumour elsewhere, especially the skin or eye. The following criteria have therefore been devised by other authors ${ }^{23}$ which afford evidence to suggest primary occurrence in the lung:

(1) No previously resected pigmented skin lesion.

(2) No ocular tumour removed or evidence of a current or previous primary melanoma in any organ.

(3) Solitary tumour in the surgical specimen from the lung.

(4) Tumour morphology consistent with malignant melanoma involving the respiratory epithelium.

(5) Junctional change, with invasion of intact bronchial mucosa by malignant melanoma cells.

(6) Full necropsy demonstrating absence of primary malignant melanoma elsewhere.

Applying these criteria to previously reported cases in the literature greatly reduces the number of "probable" primary cases of malignant melanoma of the bronchus. ${ }^{23}$ In our case the patient presented with a solitary tumour showing all the morphological and staining characteristics of a malignant melanoma together with invasion of intact bronchial mucosa and convincing clinical evidence of lack of a primary tumour elsewhere.

Why should malignant melanomas develop in the bronchi when melanocytes are not present, apparently, in the normal respiratory tract? The most likely explanation is that these tumours arise from melanocytes which have migrated along with downgrowth of the primordial respiratory tract during fetal life from the primitive foregut, somewhere between the pharynx and the oesophagus, ${ }^{2}$ two sites at which malignant melanomas are known to occur. Alternatively, it has been speculated that tumour may arise from areas of squamous metaplasia ${ }^{4}$ in which certain of the epithelial cells have undergone differentiation towards melanocytes, a phenomenon which explains the occurrence of pigmented squamous and basal cell tumours in the skin. ${ }^{56}$ Melanocytes have also rarely been identified within the basal layer of the squamous epithelium in the larynx of a patient suffering from chronic laryngitis which suggests that a similar change could occur in irritated bronchi undergoing squamous metaplasia. $^{7}$

The long term survival of patients with primary malignant melanoma is unpredictable but is usually poor. Our patient remains well at 4.5 years following resection with no recurrence or metastases, which is further evidence of the likelihood that this represents a primary tumour. However, conclusive evidence may only be obtained after necroscopic examination.

1 Salm R. A primary malignant melanoma of bronchus. $f$ Pathol Bacteriol 1963;85:121-6.

2 Jensen OA, Egedorf J. Primary malignant melanoma of the lung. Scand F Respir Dis 1967;48:127-35.

3 Carstens PHB, Kuhns JG, Cyrus G. Primary malignant melanomas of the lung and adrenal. Hum Pathol 1984;15: melano-4.

4 Taboada CF, McMurray JO, Jordan RA, Seybold WD. Primary melanoma of the lung. Chest 1972;62:629-31.

5 Reid JD, Mehta VT. Melanoma of the lower respiratory tract. Cancer 1966;19:627-31.

6 Shivas AA, MacLennan WD. "Melanogenic metaplasia" of mucous glands. Br f Cancer 1963;17:411-4.

7 Busuttil A. Dendritic pigmented cells within human laryngeal mucosa. Arch Otolaryngol 1976;102:43-4.

Thorax 1996;51:224-226

Pulmonary Division, Cliniques

Universitaires Saint-

Luc, Brussels,

Belgium

Ph Collard

D O Rodenstein

Ch Francis

Ruhrlandklinik, Center for Thoracic Center for Thery and Pulmonary Medicine, Essen, Germany

L Freitag

Intensive Care Division, Cliniques

Universitaires Saint-

Luc, Brussels,

Belgium

M S Reynaert

Correspondence to:

$\mathrm{Dr} \mathrm{Ph}$ Collard, Cliniques

Universitaires Saint-Luc,

Service de Pneumologie,

Avenue Hippocrate 10

1200 Brussels, Belgium.

Received 20 October 1994

Returned to authors

16 January 1995

Revised version received

2 February 1995

Accepted for publication

21 February 1995

\section{Respiratory failure due to tracheobronchomalacia}

\author{
Ph Collard, L Freitag, M S Reynaert, \\ D O Rodenstein, Ch Francis
}

\begin{abstract}
A case is described of tracheobronchomegaly progressing to extensive tracheomalacia, complicated by episodic choking, recurrent pulmonary infections, and irreversible hypercapnic respiratory failure. A Y-shaped tracheobronchial stent was placed endoscopically to splint the trachea open, with excellent clinical and physiological improvement. New stent designs may provide long term palliation in selected cases of diffuse tracheal collapse or stenosis, and offer an alternative to surgical repair.
\end{abstract}

(Thorax 1996;51:224-226)

Keywords: tracheobronchomalacia, tracheobronchial stent, respiratory failure.
Tracheomalacia is a condition caused by weakness of the tracheal wall due to softening of the supporting cartilages. ${ }^{12}$ Initially it causes dynamic collapse during forced expiration and cough, which can be prevented by continuous positive airway pressure (CPAP). ${ }^{3}$ In the most severe cases the trachea becomes distorted and permanently narrowed.

\section{Case report}

A 52 year old man was diagnosed as having tracheobronchomegaly in 1982. In 1983 he received prolonged assisted ventilation for acute respiratory failure from bilateral bronchopneumonia. Fibreoptic bronchoscopy showed expiratory collapse of an enlarged trachea during spontaneous breathing, but the patency was maintained with a CPAP of $10 \mathrm{~cm} \mathrm{H}_{2} \mathrm{O}$. Between 1984 and 1986 he had three further episodes of acute respiratory failure necessitating mechanical ventilation. Between 1987 and 1990 the patient practised postural drainage twice daily and his condition remained stable.

In 1991 he developed difficulty in eliminating secretions despite postural drainage and he 


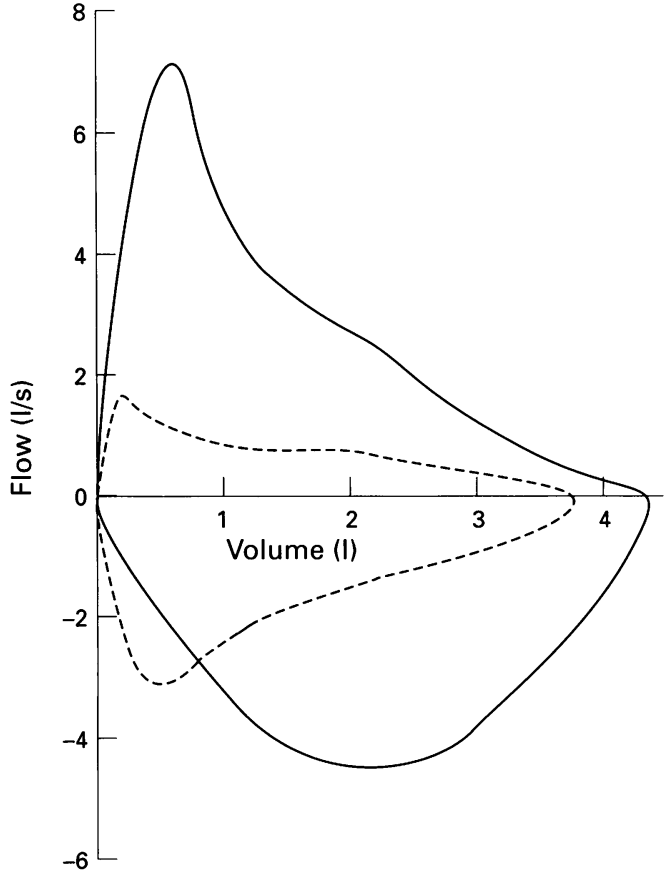

Flow-volume loop before (hatched line) and after (solid line) tracheal stenting.

required readmission for infections and distress; a further fibreoptic bronchoscopic examination revealed the trachea to be large in the lateral axis and considerably distorted and narrowed in the anteroposterior axis, with permanent collapse of its entire intrathoracic portion. The length of the stenosis was $13 \mathrm{~cm}$ with little variation during forced inspiratoryexpiratory manoeuvres. In May 1991 severe obstructive sleep apnoea developed (apnoea/ hypopnoea index of 74) and he needed a CPAP apparatus at $9-10 \mathrm{~cm} \mathrm{H}_{2} \mathrm{O}$ to relieve sleep related obstruction. His daytime condition did not improve and he required courses of intravenous antibiotic treatment for pulmonary infections. Bronchial drainage was somewhat facilitated by CPAP application during physiotherapy. A trial with oral steroids reduced the sputum quantity but pulmonary function tests were not improved.

In April 1992 he deteriorated with ongoing bronchial suppuration and severe chronic hypercapnia. He was mechanically ventilated several times; nasal access bilevel positive airway pressure (BiPAP) was ineffective in improving his arterial blood gases. In July 1992 a $14 \mathrm{~cm}$ long $\mathrm{Y}$-shaped stent was inserted into the trachea through direct laryngoscopy under general anaesthesia (Dynamic Stent, Rüsch,
Kernen, Germany). The stent was made of horseshoe-shaped steel clasps incorporated in a silicone body with a flexible posterior membrane. It was put into position with forceps, which were used to grasp the bronchial limbs from inside, and a laryngoscope. The inverted $\mathrm{Y}$ rested on the carina, its limbs extended for $2 \mathrm{~cm}$ into each main bronchus, ${ }^{4}$ and it was firmly maintained between the anterior and posterior walls of the trachea.

Breathing and sputum clearance subsequently became easy without postural drainage. The patient is fully rehabilitated into his social and professional life, arterial blood gas tensions are normal, and the pulmonary function tests now show no obstructive component and an increased vital capacity (figure). Polysomnography has confirmed the persistence of obstructive sleep apnoea and he still requires CPAP at night. He is stable 40 months after stenting (table).

\section{Discussion}

In tracheobronchomalacia excessive dynamic compression of the major conducting airways and loss of laminar airflow cause increased airways resistance and effort of breathing, prolonged expiration, and distal air trapping. Due to an inefficient cough mechanism, retention of secretions with recurrent pneumonia, bronchiectasis and lung scarring occur.

Nasal CPAP prevents dynamic collapse in patients with tracheomalacia. Intermittent CPAP during the day also results in clinical improvement with easier sputum production, reduction of medical care, and better exercise tolerance. A pressure of $10 \mathrm{~cm} \mathrm{H}_{2} \mathrm{O}$ provides optimal splinting of the airways in most patients. $^{3}$

The management of diffuse tracheal stenosis is a difficult problem. Complications and failure rates of surgical techniques tend to be unacceptably high. The use of endoscopically placed stents provides a convenient and much less invasive alternative. Expanding wire stents have been used in adults with tracheobronchomalacia and chronic respiratory insufficiency. ${ }^{56}$ However, these metal stents can cause granulomata formation, severe haemoptysis, or tracheobronchial rupture. ${ }^{6}$ Dumon's dedicated tracheobronchial stents, made of moulded silicone, have been used widely for obstruction due to tumours, but are not well suited for diffuse tracheobronchomalacia. ${ }^{7}$

The Y stent appears to be particularly good for diffuse tracheal collapse. ${ }^{4}$ The method for

Arterial blood gas tensions at room air and results of pulmonary function tests

\begin{tabular}{|c|c|c|c|c|}
\hline & Before stenting & 1 month after stenting & 1 year after stenting & 2 years after stenting \\
\hline $\mathrm{PaCO}_{2}(\mathrm{kPa})$ & $10 \cdot 0-15 \cdot 0$ & $6 \cdot 0$ & $5 \cdot 1$ & $5 \cdot 5$ \\
\hline $\mathrm{PaO}_{2}(\mathrm{kPa})$ & $4 \cdot 0-6 \cdot 7$ & $9 \cdot 1$ & $11 \cdot 3$ & $9 \cdot 1$ \\
\hline PEF $(1 / s)$ & $1.66(20)$ & $5.43(67)$ & $6.59(81)$ & $7 \cdot 16(88)$ \\
\hline $\mathrm{FEV}_{1}(\mathrm{l})$ & $1.02(24)$ & $2 \cdot 8(66)$ & $3 \cdot 12(73)$ & $3.01(71)$ \\
\hline $\mathrm{VC}(1)$ & $3.50(50)$ & $3 \cdot 73(62)$ & $4 \cdot 27(70)$ & $4 \cdot 38(72)$ \\
\hline $\operatorname{Vemax}_{50}(1 / \mathrm{s})$ & 0.75 (18) & $3.02(71)$ & $2 \cdot 47(58)$ & $2.55(60)$ \\
\hline $\operatorname{Vimax}_{50}(1 / \mathrm{s})$ & $1.55(20)$ & $3.62(47)$ & $3.91(51)$ & $4 \cdot 47(58)$ \\
\hline
\end{tabular}

Values in parentheses are percentages of predicted.

$\mathrm{PaCO}_{2}, \mathrm{PaO}_{2}=$ arterial carbon dioxide and oxygen tensions; $\mathrm{PEF}=$ peak expiratory flow; $\mathrm{FEV}$ = forced expiratory volume in one

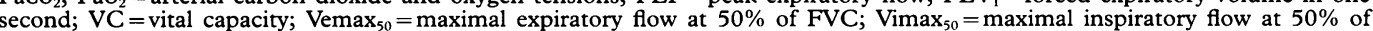
FVC. 
insertion is straightforward and minimally invasive, ${ }^{8}$ and the stent can be left in place long term without swallowing, voice, or coughing problems.

Our patient had a major and sustained improvement of his lung function with disappearance of his airways obstruction. We believe that restoration of airflow was the major factor in this clinical improvement. He still has copious purulent secretions colonised by Pseudomonas due to bilateral bronchiectasis, but eliminates secretions easily and infection is no longer a problem.

1 Meyer E, Dinkel E, Nilles A. Tracheobronchomegaly: clinical aspects and radiological features. Eur 7 Radiol 1990;10: aspects
2 Woodring JH, Howard RS, Rehm SR. Congenital tracheobronchomegaly (Mounier-Kuhn syndrome): a report of 10 cases and review of the literature. $\mathcal{F}$ Thorac Imaging 1991 6:1-10.

3 Ferguson GT, Benoist J. Nasal continuous positive airway pressure in the treatment of tracheobronchomalacia. $\mathrm{Am}$ Rev Respir Dis 1993;147:457-61.

4 Freitag L, Eicker R, Linz B, Greschuchna D. Theoretical and experimental basis for the development of a dynamic airway stent. Eur Respir $\mathcal{F}$ 1994;7:2038-45.

5 Nashef SAM, Dromer C, Velly JF, Labrousse L, Couraud L. Expanding wire stents in benign tracheobronchial disease: indications and complications. Ann Thorac Surg 1992;54: $937-40$

6 Rousseau H, Dahan M, Lauque D, Carré P, Didier A, Bilbao I, Herrero J, Blancjouvant F, Joffre F. Self-expandable prostheses in the tracheobronchial tree. Radiology 1993; 188:199- 203.

7 Dumon JF. A dedicated tracheobronchial stent. Chest 1990; 97:328-32.

8 Freitag L, Tekolf E, Greschuchna D. Development of a new insertion technique and a new device for the placement of bifurcated airway stents. Surg Endosc 1994;8:1409-11.

\title{
Commentary: yet more to see down the bronchial tree?
}

Departments of Thoracic Medicine, Whittington Hospital and University College London Hospitals London, UK M R Hetzel

\author{
Martin R Hetzel
}

These three case reports illustrate the range of rare conditions that may be encountered from time to time at diagnostic bronchoscopy. Many may at least superficially resemble bronchial carcinomas, but lead to surprises when full laboratory results become available from bronchoscopic biopsy samples. The case described by Collard et al illustrates the usefulness of direct inspection at bronchoscopic examination in evaluating the dynamics of the airway wall.

In the case of pulmonary actinomycosis described by Hsieh et al bronchoscopic examination gave the diagnosis by demonstrating a cavitating lesion which could be entered with the bronchoscope and provided positive microbiology samples. Interestingly, however, the authors were cautious in making a diagnosis of actinomycosis alone and were concerned at the possibility of a fungal infection - presumably because of the radiographic characteristics of the meniscus sign and the previous diagnosis of old tuberculosis.

Pulmonary actinomycosis is very rare and many cases are only diagnosed retrospectively from resected specimens (sadly the author's personal experience!) unless stronger clues such as sinus formation onto the chest wall with characteristic sulphur granules are present. The most common misdiagnosis is of bronchial carcinoma and, while some patients may not suffer too much if a resected "cancer" turns out to be actinomycosis, it is of course a tragedy if this rare but treatable disease is completely missed. Confusion with tuberculosis is a further problem, both from the radiological appearance and because Actinomycosis israelii can stain acid fast. Moreover, some reports have described cases of colonisation of tuberculous cavities Actinomycosis is more common in the lower lobes but upper lobe lesions occur and are then more likely to mimic tuberculosis. In the case described here one presumes that the initial diagnosis of tuberculosis was erroneous. It is interesting to note that rifampicin has been tried in the treatment of actinomycosis ${ }^{1}$ so, if this drug was included in the three-drug regimen used, it might have held the disease under partial control during the previous period of empirical treatment for tuberculosis.

This patient was susceptible to pulmonary actinomycosis from two risk factors - namely, dental sepsis and diabetes. Because of the anaerobic conditions required for actinomycosis to flourish, response to penicillin and other appropriate antibiotics tends to be slow. Surgery alone is unlikely to cure, although surgical drainage is usually recommended where there is abscess formation. Some cases are cured by antibiotic treatment alone. In this case the authors imply that they elected for immediate surgery because of a tentative diagnosis of fungal infection although, somewhat paradoxically, they actually had proof of actinomycosis from their bronchoscopic samples. One might speculate on the possibility of curing this patient with prolonged antibiotic therapy and postural drainage. However, because of the possibility of a chronic infection in a diabetic subject, sacrificing some viable lung at lobectomy was probably still the best management option.

As reported by Farrell et al, primary malignant melanoma of the bronchus is very rare and many previous case reports have not fully 